\title{
Study On the Frame Design of Requirements and Functions of the Assistance Decision-making Support System for Smoke Screen Support \\ Cheng Cui ${ }^{1, a}$, Xuzheng Zhu' ${ }^{2, b}$, Weizhao Yao ${ }^{3, c}$, Sheng $\mathrm{Xu}^{4, \mathrm{~d}}$, Kunlin $\mathrm{Nie}^{5, \mathrm{e}}$ Institute of NBC Defence, Beijing, 102205, China \\ eemail542112706@qq.com
}

\begin{abstract}
Keywords: Smoke Screen Support; Assistance Decision-making; Optimal Allocation and Deployment; Situation monitoring and surveillance
\end{abstract}

\begin{abstract}
This paper builds up the basic framework of the Assistance Decision-making Support System for Smoke Screen Support by analyzing the Requirements and Functions of the Assistance Decision-making Support System for Smoke Screen Support and the auxiliary decision-making procedure for typical combat patterns relating to smoke screen support, in an attempt to provide functional solutions for the problems such as the optimization of allocations and deployment of smoke-generating forces, the early-warning to airborne situations, the analysis of threats, the control and discharge of smoke, the evaluation and correction of smoke screen generation so as to realize the integration and coordination, comprehensive command and control of the airborne smoke spraying system, smoke vehicles and smoke pots.
\end{abstract}

\section{Introduction}

Modern smoke screen has unique advantages in terms of its capability of resistance to precision guided weapons and observation and aiming weapons. Along with the rapid development of photoelectric weapons and air defense operations, the armed forces of PLA has paid more attention on the special function of smoke screen support in the area of air defense of photoelectric weapons in the joint operations. Some troops have already been equipped with newly-produced smoke generation equipment, while the other will be equipped with such devices. However, it is imperative to answer the question, "How will the battle effectiveness of the forces equipped with such smoke generating devices come into being?"

\section{The Analysis of the Requirements on the Assistance Decision-making Support System for Smoke Screen Support}

From the perspective of system engineering, "requirements" are the relevant descriptions of the types of the system, its functions, structure, performance, movement and quantity, which are essential to the realization of the preset goals and settlement of matters in real operations. The analysis of the requirements aims to provide clear and complete information on what the users would use to the decision makers. Likewise, the analysis of the requirements on the assistance decision-making support system for smoke screen support aims at clarifying the goals of the assistance decision-making support system, the fundamental functions, the basic data and models, techniques for the smoke support decision making process, etc.

\section{Goal Analysis}

The goal of the assistance decision making for smoke screen support is to achieve the ultimate goal of the relevant military operations by employing the assistance decision making system for smoke screen support. As a whole, such a goal is to automatically capture a variety of airborne information for information integration and information exchange by using the assistance decision making system in wartime. It is to make judgment on the threats of the incoming target by a series of model calculation. Based on the quantities and performance of the equipment in service and the combination of smoking generating vehicles and pots, a network of air-to-ground smoke screen support will be formed. By using simulation imitation, the relevant allocation and deployment plans 
will be optimized. Thus, a final smoke screen support plan can be made, which will be sent to each smoking generating team by smoke spraying control command instructions. In this way, the intelligentized assistance decision making for smoke screen support command under informationization conditions can be achieved. Consequently, the survival and capabilities of equipment in the system-to-system resistance can be improved.

Particularly, prior to each auxiliary decision of smoke support, the goal of smoke support must be clarified. Normally, there are many goals ranging from concealment of a fixed ground target, covering the maneuver of troops, ground targets, protecting a command post, an airport, ports, missile launching silos, and artillery positions. Therefore, the support approaches vary from goal to goal. Take a command post as an example, for one thing, one must make sure the command post will be not be struck by enemy's fire, for another, one must make sure the communication of the command post will not be interfered by the smoke screen generated by our own team. In addition, one must identify the striking means or reconnaissance means likely to be used by the enemy for providing the corresponding smoke screen support. Since missiles, precision guided bombs, bombers, artillery has different striking features, the commander must provide the relevant smoke support in response to their own features. It is unpractical to use one way for all.

\section{The Analysis of Functions}

The analysis of functions of the assistance decision making system for smoke screen support is to analyze "what can the system do?" or "what kind of service can the system provide to its users?" In terms of smoke screen support, the roles of the assistance decision making system can play as the below.

1) Integrating and Processing the Battlefield Information

Battlefield is a complex and dynamic environment. To make a right decision, one must have a good command of the situation on the battlefield. Therefore, one of the important functions of the assistance decision making system for smoke support is to continuously process and integrate a variety of information related to the battlefield on the basis of perception of the situations of the battlefield and literally demonstrate the integrated information to the decision maker for making judgment. Take the airborne information as an example, what the air defense troops has provide to us is nothing but a series of data such as the types of incoming targets, its speed, height and direction, and so on. Such data cannot be literally used by smoke screen support commanders. The point is how to make judgment on the data and integrate them with smoke support decision making. That is exactly what the system is designed to do. The system can retrieve various correlated data and threat-judging models and prejudge the threats of incoming target, the means of guidance, its trajectories and striking means and etc. Further, it can infer its striking target, time, threats, the chances of using smoke screen and the effectiveness.

2) The Making of Smoke Screen Support Plan

The nucleus of the assistance decision making of smoke support is to provide assistance to the commander to make judgment upon the smoke support. The smoke screen support plan can be divided into two parts: First, at the early stage it can provide assistance to the commander to optimize the deployment of smoke generating teams at their position and further make allocation plans for the teams. Second, when receiving airborne early warning, it should be able to help the commander make a smoke spraying program to provide smoke screen support based on the real situation. The allocation and deployment plans of smoke teams and the smoke spraying program both come out of the perception of the commander on the relevant situation, which are also the operation-oriented decisions based on the comprehensive analysis and processing of the information on the battlefield, air, enemy, suppliable troops.

3) The Function of Smoke Screen Support Calculation

Calculation mainly indicates that the system can help staff officers to make various calculations about the smoke support such as the proportions of smoke pots, smoke generating vehicles and aerial smoke discharging system, about the interval of allocations, the quantity of aerial smoke projectiles, the direction of smoke screen, the starting time and end time, the type and quantity of smoke agent, the quantity of fuel, the quantity of the aerial smoke discharging projectiles, the waves 
of aerial smoke, the intervals, emitting angle and height, and so on. By using various built-in models in the assistance decision making system and typing the current information about the threatening target, protected target, smoke generating equipment and the meteorological and geographical information, the commander can obtain the result automatically, which can provide references for making specific smoke discharge program and lay a solid foundation for the coming smoke emission and control.

4) The Function of Deduction and Simulation of Smoke Support

The function of deduction and simulation of smoke support of the assistance decision making system indicates that it is capable of correcting and optimizing the smoke screen support by using the principles and approaches of Operation Research and System Engineering and computer simulation technology, imitating the process and result of the allocation and deployment plan of smoke generating teams and the smoke discharging program and further evaluating the result. In this way, one can carry out thoughtful and reasonable investigations into different plans and programs and fully inspect the interferences stemming from different factors. This approach is far better than the result from static evaluation and can raise the scientific and rational level of the plans and programs.

5) The Function of Integrative Control on Smoke Generating Elements

One of the important functions of the assistance decision making system for smoke screen support is to achieve the integrated control of various smoke generating elements and command and control the subordinate smoke elements by voice, telegraph and digital data link and so on. Based on the previous calculations, one can formulate smoke discharge and control instructions/commands such as discharging means, the types of the smoke screen and duration, and issue the instructions to each smoke generating element. This function can help avoid separate operations and really form an entire network of smoke screen support to protect the relevant target.

6) The Function of Evaluating Smoke Screen Efficiency

The evaluating function of the system indicates such a system enables the commander to carry out real-time monitoring and surveillance on the process of smoke screen support and its efficiency. By using this function, the commander can grasp the real process and find out problems such as the incomplete concealment, the improper overlapping parts of smoke screen and unfavorable integration in time. The evaluating function can provide the commander with the efficiency of smoke screen plans, particularly whether the efficiency has already reached up to the expected goal with the change of environment and can give suggestions to the commander for adjustment to make sure the achievement of the goal.

\section{The Analysis of the Auxiliary Decision-making Procedure for Typical Combat Patterns of Smoke Screen Support}

As the last step of air defense, smoke screen support is provided mainly in response to two air defense combat patterns. One is for typical anti-air-raid operations, the other for typical anti-reconnaissance operations. The typical anti-air-raid operations are mainly conducted in defense against aerial threats from a variety of precision guided weapons and bombers by concealing a target or a landmark with smoke screen or by interfering with the trajectory of incoming weapons or by interfering with the guidance of enemy's precision guided weapons so that such weapons can lose their target or deviate from it, or by exposing a fake target to the coming bombers and concealing the primary one so that the enemy would make misjudgment on the target, aiming to improve the survival of the target. The typical anti-reconnaissance operations are mainly conducted in defense against the reconnaissance in the air or space. Smoke screen can reduce the detecting distance of the reconnaissance by visible light, the infrared reconnaissance or the microwave detection so as to impede the enemy's reconnaissance and thus disable the enemy to acquire the information about the target.

Actually, the auxiliary decision of smoke screen support is a supplement to the decision-making ability. One of the priorities of a typical decision-making system is to perceive, acquire, integrate and process battlefield information. Based on the result and the evaluation and judgment out of the 
knowledge base on the battlefield situation, the commander will choose a proper operation plan, that is to say, make a final decision and provide the decision for the decision maker for implementation. The implementing process will bring new changes to the battlefield and may transform the battlefield situation and force the decision maker to make some adjustment.

On that principle and based on the features of typical smoke screen support operations and the fundamental decision making procedure, the auxiliary decision-making process can be indicated in the figure 1 .

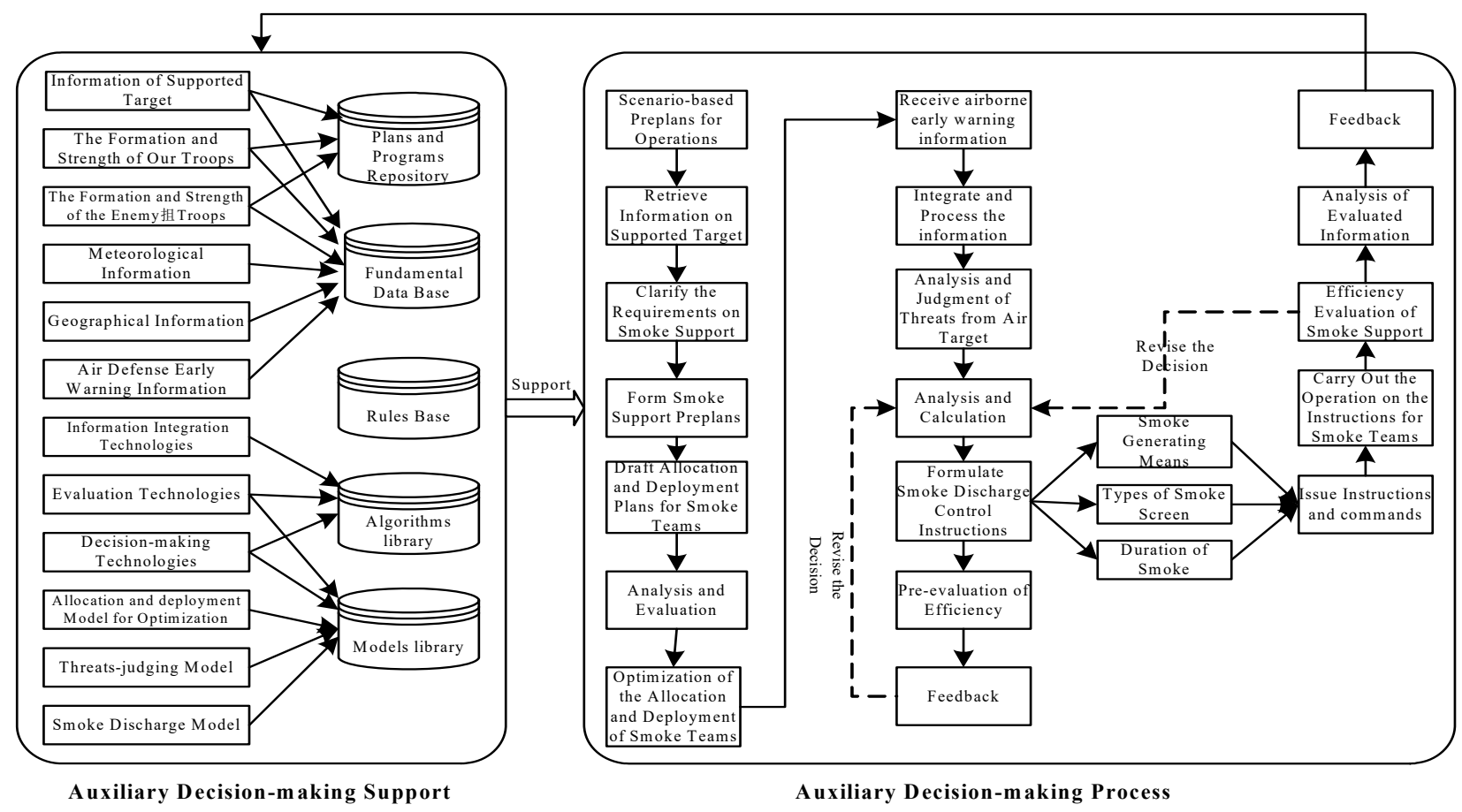

Figure 1. The auxiliary decision-making process typical smoke screen support

Above all, decision makers should make scenario-based preplans for operations in accordance with the entire situation on the battlefield, military intentions and the comparative analysis of the military strengths of two sides. Starting from the preplans, decision makers should pose the relevant information on smoke screen support and the relevant requirements on smoke support, including the scope, the quantity of targets, geographical location, the main source of threats, the striking means likely to be used by the enemy, reconnaissance, the direction of incoming weapons, the guidance of the weapons, the powers of the strike, the reconnaissance trajectory, etc. Smoke Screen support preplans can be made based on the information. In accordance with the standard for the defense of Maximum Striking Power, decision makers make up allocation and deployment programs for smoke teams and by using different calculating methods optimize the smoke teams so that the smoke vehicles and smoke pots, and aerial smoke discharging systems can form a rational network topology structure, which can be complementary to one another, forming an air-to-ground defense system of smoke screen.

After the pre-allocation and pre-deployment of smoke forces has been finished, each smoke discharge team is on standby at their position, continuously receiving information. Once they receive the airborne early warning given by their commander, they will take such information into account as local weather condition, geographical situation and further integrate and process all the information and make judgment on the air target and get ready for providing smoke support. Based on the calculation and analysis of smoke discharge control and the prediction of efficiency of smoke screen, decision makers make up specific smoke discharge control instructions, including the discharging methods, duration, categories of smoke screen and have the instructions and commands issued and sent for implementation.

After implementation, by monitoring, analyzing and evaluating the efficiency of smoke screen on the real battlefield, decision makers can find out the shortcomings and defects coming up in the 
process of smoke generation and make adjustment and remedies and send feedback to various knowledge bases to improve the plans and programs repository of the auxiliary decision making for smoke support and make it more rational.

\section{The Basic Framework of the Assistance Decision-Making System of Smoke Screen and Its Main Functional Modules}

With modern information technology as its main means, the assistance decision-making system makes full use of a wide range of scientific knowledge in the domains including database, computer, artificial intelligence, system engineering, operational research, management science and provides its users with a fast and efficient human-machine interaction system for making a right decision in response to a specific matter by offering a series of processes such as essential prerequisites, background parameters and model calculation and so on. As a sort of human-machine interaction system, it enables the commander to make analysis and judgment on the situation of the battlefield and finish a series of model computing process and provide the commander with assistance for making a fast, scientific and rational plan for smoke support.

\section{The Basic Framework}

As an integrated system supported by many technologies and functional modules, the assistance decision-making system for smoke support mainly consists of a series of subsystems such as database, model library, algorithms library, plans and programs base, rules base, information processing, communication, weapons control, man-machine interaction and graphical interface, etc.

Functionally, the assistance decision-making system for smoke support can be divided into application level, decision-making level, management level, decision-making support level, information support level. Its framework is indicated in figure 2.

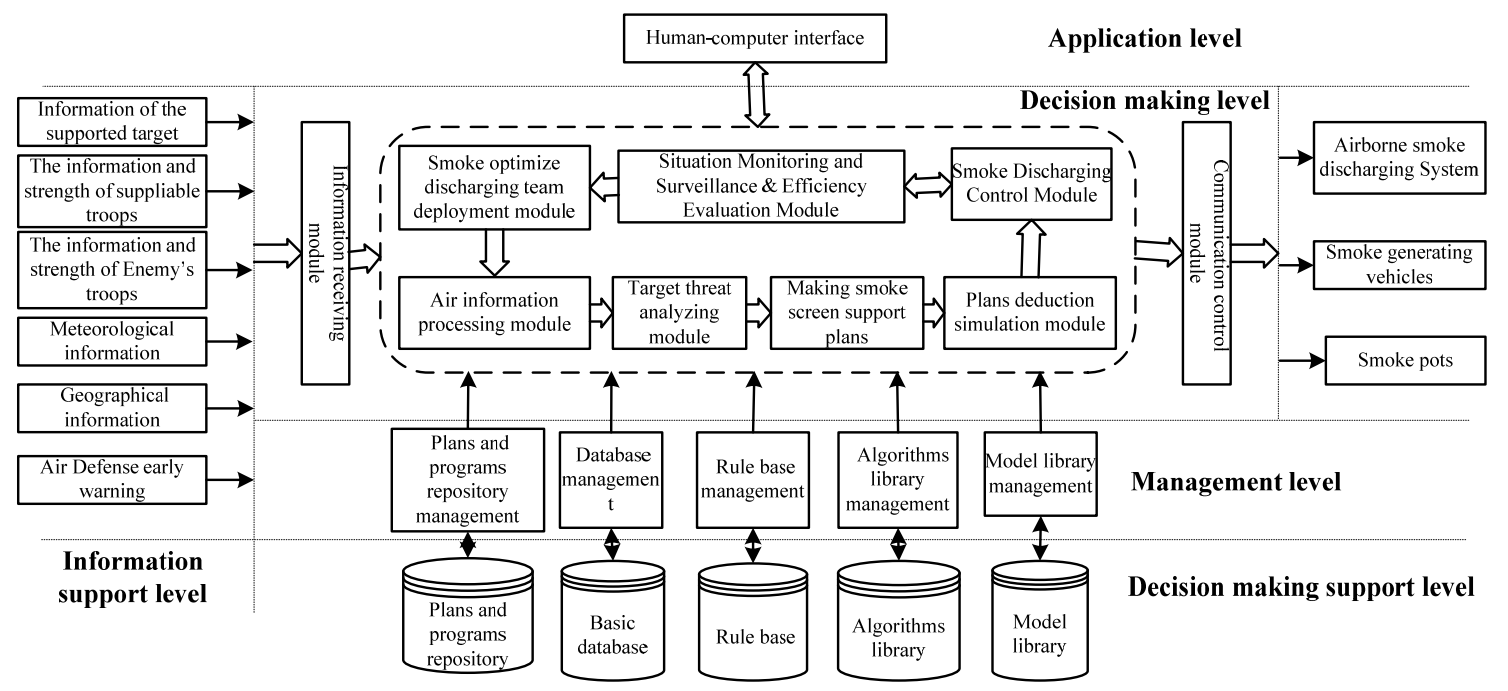

Figure 2. Framework of the assistance decision-making system for smoke support

1) Application level

As the interactive medium between a commander or decision maker and the assistance decision-making system, application level mainly include human-computer interface through which the commander can interfere in the decision-making process of the system and duly adjust the information input of the system for a better decision and accept the information output of the system like the making of a plan or various instructions.

2) Decision-making level

As the nucleus of the entire assistance decision making system, decision making level is designed to process a variety of knowledge and deduce information from the knowledge and provide assistance to the commander for making decisions. It comprises a series of functional modules such as smoke discharging team deployment module, air information processing module, target-threat analyzing module, plans -deduction simulation module, smoke discharging control module, situation monitoring and surveillance $\&$ efficiency evaluation module, etc. 
3) Decision support level and management level

Decision support level of the assistance decision-making system for smoke support includes the basic database, plans and programs repository, rules base, algorithms library and model library, which are being used in the decision-making process to provide essential data, calculations, models, rules and so on. All of these are the basis of decision making and inference. Management level is designed to set up, revise, add and delete the information in database, plans and programs base, rules bases, algorithms library and model library.

4) Information support level

Information support level encompasses various preset information for making decision such as the information of the supported target, the information and strength of PLA troops, the information and strength of Enemy's troops, meteorological information, geographical information, air defense early warning information, etc.

\section{Smoke discharging team deployment optimization module}

Abiding by the optimal procedure of smoke discharging team deployment, the module optimizes the deployment of smoke forces and obtains the best deployment plans on the basis of enemy's air raid main attack judgment model and smoke support scope calculation model so as to achieve the maximum survival of the supported target.

\section{Air information processing module}

On the principle of "real-time gathering, multi-channel transmission and integrative process", this module include the communication module and interface linked with air defense early warning particularly for receiving early warning information from the higher commands. It also includes a variety of meteorological and geographical information sensors for collecting the relevant information in a small area. In addition, it also has an information integration function for receiving and collecting various air early warning information, geographical information, meteorological information and the information related to both sides engaged in the battle so as to process all the information and further make a situation map to provide information for making decisions.

\section{Target threat analysis module}

On the basis of the destructive power of incoming target and its mobility and intention, this module mainly makes full use of target integration data, evaluates the incoming target without stop, judges the levels of the coming threats and makes a final judgment for providing smoke support.

\section{Smoke Discharging Control Module}

In accordance with the deployment of smoke forces and threat analysis of air target and a series of model calculations, this module's main function is to provide the information such as assignment of tasks of smoke teams, the time for generating smoke, the quantity of smoke. Besides, it also helps make up plans for smoke support and issue instructions to each smoke team for further action.

\section{Situation monitoring and surveillance $\&$ efficiency evaluation module}

Situation monitoring and surveillance and efficiency evaluation module mainly enables a commander to monitor the real-time situation in the process of generating smoke and making prompt and scientific evaluation on the efficiency of smoke screen support. Among other things, situation monitoring and surveillance includes various voice, file, telegraph commands to be used in the operations, and videos, images and icons to demonstrate the ongoing operation, which will be helpful for the commander to grasp the progress of smoke support at any time. Evaluation on the efficiency of generated smoke enables a commander to evaluate the efficiency of the smoke screen. By evaluation, the commander can judge the effectiveness of smoke screen on the battlefield so that the commander can make adjustment and revise the smoke support plan for higher efficiency.

\section{Conclusion}

The paper analyzes the military requirements on the auxiliary decision making of smoke screen support and explores into the fundamental procedure of the assistance decision making of smoke screen support and sets up the main functional framework of the assistance decision making of smoke screen support and puts forward the requirements upon the auxiliary decision-making functions such as the optimization of smoke screen generating forces, airborne early warning, threat 
analysis, the control of smoke discharge, evaluation on the efficiency of generated smoke and the relevant correction. The paper provides direction for the modeling of assistance decision making of smoke screen support.

\section{References}

[1] Lei Cao, Guangyu Bao, Guoyou Chen. Command and Information System[M].Beijing: Industrial Press of National Defense ,2012:77.

[2] Liyong Wang. Research on the Command and Information System of the Army[M].Beijing: Publishing House of the University of National Defense: 2007.

[3] Xueshan Luo. Analysis and Design of Command and Information System[M].Changsha: Publishing House of the Science and Technology University of National Defense, 2006.

[4] Fuyuan Li, Hua Li. The Trend of Command and Control of Operations in the Age of Information [J].Firepower and Command and Control, 2008.12,33(12):2-4.

[5] Ke Zhang, Yongcai Liu, Shiyi Guan. A Study of Missile Attack and Defense Simulation towards the Efficiency and Evaluation of Auxiliary Decision-making Support System [J] Tactical Missile Technology, 2004.5,(3):54-56. 